Dieses Dokument ist eine Zweitveröffentlichung (Postprint) /

This is a self-archiving document (postprint):

Carlos Sánchez-Sánchez, Adrien Nicolaï, Frédéric Rossel, Jinming Cai, Junzhi Liu,

Xinliang Feng, Klaus Muillen, Pascal Ruffieux, Roman Fasel, Vincent Meunier

On-Surface Cyclization of ortho-Dihalotetracenes to Four- and SixMembered Rings

Erstveröffentlichung in / First published in:

Journal of the American Chemical Society. 2017, 139 (48), S. 17617-17623. ACS Publications. ISSN 1520-5126.

DOI: https://doi.org/10.1021/jacs.7b10026

Diese Version ist verfügbar / This version is available on:

https://nbn-resolving.org/urn:nbn:de:bsz:14-qucosa2-365757 


\title{
On-Surface Cyclization of ortho-Dihalotetracenes to Four and Six-membered Rings
}

Carlos Sánchez-Sánchez, ${ }^{1,2^{*}}$ Adrien Nicolaï, ${ }^{3+}$ Frédéric Rossel, ${ }^{1}$ Jinming Cai, ${ }^{1 \#}$ Junzhi Liu, ${ }^{4}$ Xinliang Feng, ${ }^{4}$ Klaus Müllen, ${ }^{5}$ Pascal Ruffieux, ${ }^{1}$ Roman Fasel ${ }^{1,6}$, and Vincent Meunier ${ }^{3}$

${ }^{1}$ Empa, Swiss Federal Laboratories for Materials Science and Technology, Überlandstrasse 129, CH-8600 Dübendorf, Switzerland.

2 Instituto de Ciencia de Materiales de Madrid (ICMM-CSIC), Sor Juana Inés de la Cruz 3, 28049 Madrid, Spain

${ }^{3}$ Department of Physics, Rensselaer Polytechnic Institute, Troy, 12180 New York, USA

${ }^{4}$ Center for Advancing Electronics Dresden \& Department of Chemistry and Food Chemistry, Technische Universität Dresden, D-01062 Dresden, Germany.

${ }^{5}$ Max Planck Institut for Polymer Research, Ackermannweg 10, 55128 Mainz, Germany.

${ }^{6}$ Department of Chemistry and Biochemistry, University of Bern, Freiestrasse 3, CH-3012 Bern, Switzerland

\begin{abstract}
We report on the surface-catalyzed formal $[2+2]$ and $[2+2+2]$ cycloadditions of ortho-activated tetracene species on a $\mathrm{Ag}(111)$ substrates under ultrahigh vacuum conditions. Three different products are obtained: tetracene dimers, trimers, and tetramers. The former results from four-membered ring formation while the other two arise from cyclization toward six-membered rings. These on-surface reactions have been monitored by scanning tunneling microscopy and rationalized by density functional theory calculations. Our approach, based on the reaction of ortho-dihalo precursor monomers via formal cycloadditions, establishes a new tool for the highly active field of on-surface synthesis and provides access to novel 1D and 2D covalent carbon nanostructures.
\end{abstract}

Keywords: on-surface synthesis, cycloaddition, aryne, scanning tunneling microscopy, density functional theory

\section{Introduction}

Cycloaddition reactions are of paramount importance in today's organic chemistry and chemical industry. They are involved in the fabrication of products ranging from chemicals and medicines to optical materials ${ }^{1}$. Cycloaddition encompasses a wide variety of synthetic processes yielding, for example, three-, four-, five and six-membered rings by the reaction of carbenes with olefins, dimerization of olefins, as well as Huisgen and Diels-Alder protocols, respectively (see, for example, references ${ }^{1-4}$ for an overview on the current status of cycloaddition reactions).

Despite being an important synthetic tool of chemistry, cycloaddition reactions have received consid- 
erably less attention in the context of on-surface synthesis. The latter rests upon the adaptation of traditional solution-based chemical reactions for the fabrication of new (macro)molecular architectures under ultra-high vacuum conditions (UHV) using the catalytic properties of (mostly metallic) substrate surfaces ${ }^{5}$. Some of these reactions have successfully been explored on surfaces under UHV conditions. This is the case of the Diels-Alders reaction, ${ }^{6}$ and, more recently, of the 1,3-dipolar cycloaddition ${ }^{6,7}$ (Huisgen reaction) and the $[2+2+2]$ cyclotrimerization of alkyne groups. ${ }^{8-11}$ Among them, [2+2] cycloadditions are highly interesting as they allow the formation of cyclobutadiene moieties. A [2+2] cycloaddition has recently been suggested to be involved as an intermediate step in the thermal formation of complex structures such as substituted porphyrins or phthalocyanine tapes. ${ }^{12,13}$ It has also been proposed as the mechanism responsible for the photopolymerization of $C_{60}$ molecules on calcite. ${ }^{14}$ Graphene-like nanoribbons embedded in carbon tetragons and octagons have been recently reported, former being the indirect result of octagonal rings. ${ }^{15}$ Additionally, formation of cyclobutadiene "bridges" - resulting from a [2+2] cycloaddition of arynes - has been reported as a product of the cyclization of enediynes, or even after dehydrogenative homocoupling of pentacene molecules on $\mathrm{Au}(110){ }^{6,17}$ Furthermore, the introduction of cyclobutadiene units into ultra-narrow zigzag graphene nanoribbons (ZGNRs) has been theoretically proposed to create spin switches. ${ }^{17}$

Herein, we describe the on-surface synthesis of molecular dimers, trimers, and tetramers via formal $[2+2]$ and $[2+2+2]$ cycloadditions of tailored halogen-functionalized tetracenes on $\mathrm{Ag}(111)$ under UHV conditions. So far, on-surface aryl-aryl coupling reactions of halogen-functionalized precursor monomers have exclusively relied on the functionalization at para and meta positions. ${ }^{18}$ However, our novel design of a precursor monomer with halogen ortho functionalization allows linking activated acenes in an efficient and controlled way to obtain four- and six-membered carbon rings at predetermined positions. Specifically, we have used 2,3-dibromotetracenes where the halogen atoms are located at ortho positions. ${ }^{19}$ We have characterized the on-surface synthesis of tetracene dimers, trimers, and tetramers by means of high-resolution scanning tunneling microscopy (STM) and rationalized our experimental results with the help of density functional theory (DFT) calculations.

\section{Results}

Figure 1 a shows an STM image of the $\mathrm{Ag}(111)$ surface after deposition of a submonolayer coverage of 2,3-dibromotetracene (1) on the substrate held at $430 \mathrm{~K}$. At this temperature, three different coexisting molecular structures, arranged into highly-ordered self-assembled islands, can be clearly distinguished, namely the structures labeled 2, 3, and $\mathbf{4}$. According to their shape and dimensions, they can be assigned to the formation of dimers, trimers, and tetramers, respectively, formed upon activation of the

monomer 1 via thermally induced dehalogenation. A scheme of the proposed synthetic path to the different structures is shown in Figure $1 \mathrm{~b}$. According to this model, once the molecules are thermally 
activated at ortho positions, an aryne is formed (1'). This aryne may take one of three possible resonance structures, as will be discussed below. If two/three arynes (1') react through a formal [2+2] / $[2+2+2]$ cycloaddition, dimers (2) and trimers (3) are formed, respectively. The unexpected structure $\mathbf{4}$, however, must correspond to the tetramerization of four arynes via successive single $\mathrm{C}-\mathrm{C}$ bond formation steps. Besides the abovementioned structures 1-4, we occasionally also observe the formation of minority species such as 2,2'-bitetracenyl ("shifted dimers"). The formation of these oligomer structures can, in principle, be explained by two mechanisms. On the one hand, they can occur due to the presence of monobromo-tetracene impurities in the molecular powder used for the deposition. On the other hand, they can also appear during the on-surface synthesis step as a consequence of the formation of a $2,2^{\prime} \mathrm{C}-\mathrm{C}$ bond between monomers that are axially offset (shifted) with respect to each other. In such a situation, it is energetically too costly to rotate one of the monomers around the formed $\sigma$ bond in order to allow for the formation of a straight cycloaddition dimer $\mathbf{2}$, as we will discuss below.

(a)

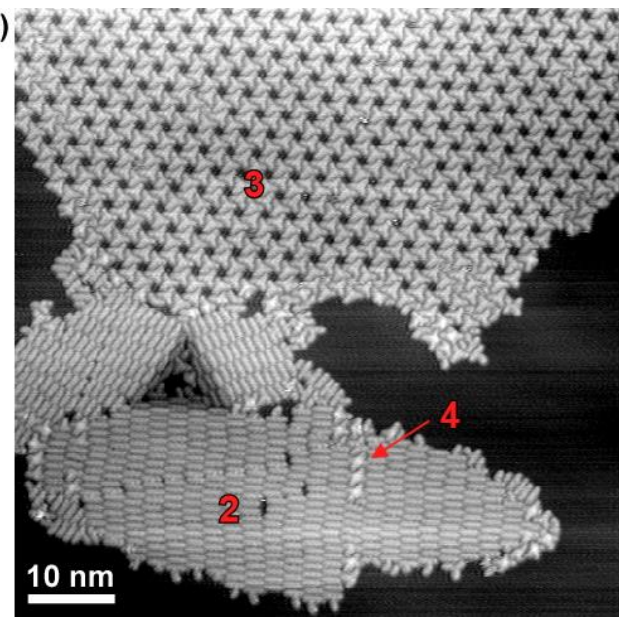

(b)

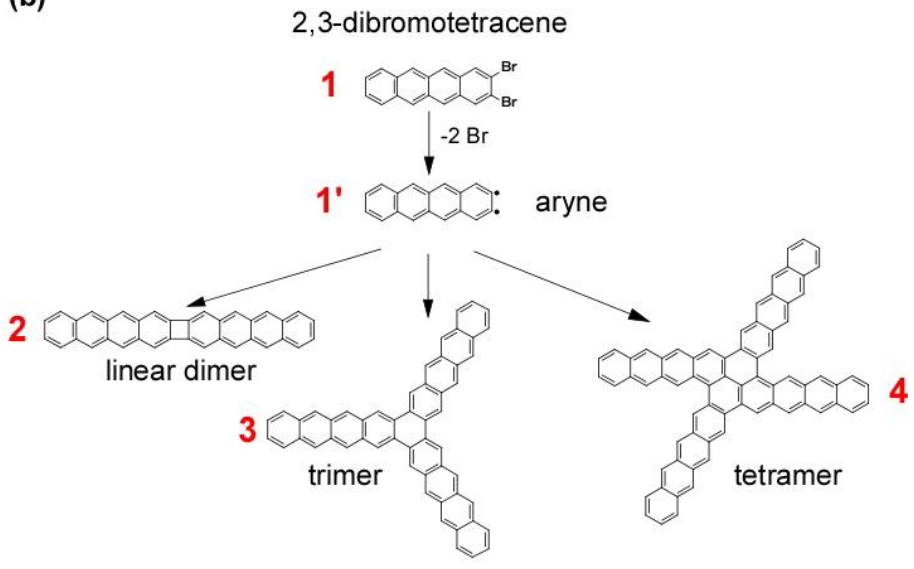

Figure 1.- Structures formed after deposition of 2,3-dibromotetracene precursor monomers 1 onto a $\mathrm{Ag}(111)$ substrate held at $430 \mathrm{~K}$. (a) STM image showing the coexistence of three different structures, labeled 2, $\mathbf{3}$ and $\mathbf{4}$. The numbers in red refer to the structures in panel (b). Vs $=1.0 \mathrm{~V}, \mathrm{I}=0.07 \mathrm{nA}, 30 \mathrm{~K}$. (b) Reaction scheme toward linear [2+2] cycloaddition dimers $(2),[2+2+2]$ cycloadditions trimers (3) and tetramers (4) from precursor (1) via the dehalogenated aryne intermediate (1'). Hydrogen atoms have been omitted for clarity.

In order to corroborate the assignment of the different structures, we have compared experimental high-resolution STM images (Fig. 2a) with STM simulations (Fig. 2c) based on DFT calculations (for details, see the Supplementary Information). The first panel of Figure 2a shows a STM image of an area labeled 2 in Fig. 1, where linear, bar-like structures surrounded by smaller dots can be discerned. The assignment of these linear structures to tetracene dimers $\mathbf{2}$ is supported by the good agreement with the STM simulation of dimers formed upon [2+2] cycloaddition (Figure 2c, left). Dots appearing between the dimers are assigned to $\mathrm{Br}$ atoms, as will be discussed below. Furthermore, comparison of the characteristic appearance of the occupied states with the STM simulation and the atomic configuration 
allows for an unambiguous assignment of the central dot to the position of the central cyclobutadiene moiety formed upon [2+2] cycloaddition. The cycloaddition products 2 are found to self-assemble into well-ordered islands with their long axis along the $\langle\overline{1} 01\rangle$ direction and exhibiting a rectangular unit cell $\left(\sim 4.7 \times 1.0 \mathrm{~nm}^{2}\right)$. This observation is in good agreement with the DFT-optimized structure for close-packed dimers stabilized by $\mathrm{Br}$ atoms (Figure $2 \mathrm{c}$ left and Fig. S3). In the case of $\mathbf{3}$, trimers exhibit a star-like shape with the three tetracene "arms" forming a $\sim 120^{\circ}$ angle with respect to each other (Fig. $2 \mathrm{a}$, middle). This is in good agreement with the optimized structure of a trimer formed upon $[2+2+2]$ cycloaddition, as seen by superimposing the optimized model (Fig. 2b, middle) on the experimental STM image (Fig. 2a middle). Further confirmation is obtained by comparison of the occupied states STM image with the STM simulation (Fig. 2c middle), where a maximum at the star center can be observed. In this case the trimers, due to their three-fold symmetry, prefer to arrange into a hexagonal unit cell, as highlighted in red in Figure $2 \mathrm{c}$ middle. Finally, we consider the special case of the tetramers 4. This unique minority phase is the result of the interconnection of four arynes into a X-shape structure upon monomer activation. One possible mechanism of formation is the bonding of two partially reacted rotated dimers $\left(\mathbf{2}^{\prime}\right)$ including the release of two $\mathrm{H}$ atoms, as schematically shown in Fig. S4 in the Supporting Information. Alternatively, a shifted dimer (2"' in Fig. S4) could react with two arynes (1') to form the final tetramer under release of two hydrogen atoms. We note that in both hypothetical pathways the starting point for the formation of $\mathbf{4}$ is the presence of an incompletely reacted dimer. As can be seen in Fig. 2 right, there is a good agreement between experiment and theory, where tetramers arrange into small islands with a rectangular unit cell. 
(a)

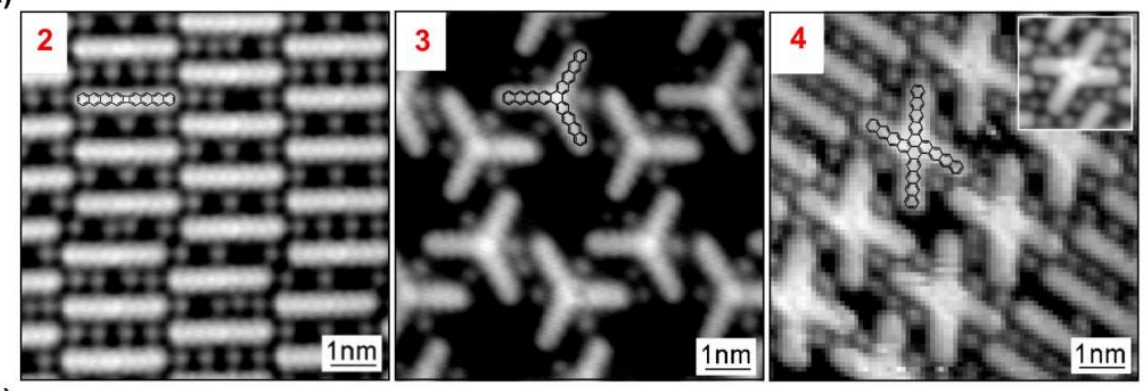

(b)

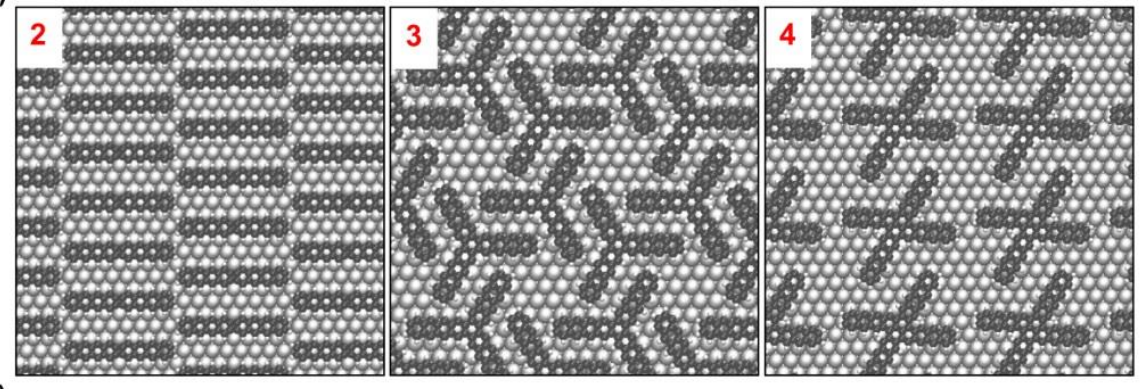

(c)
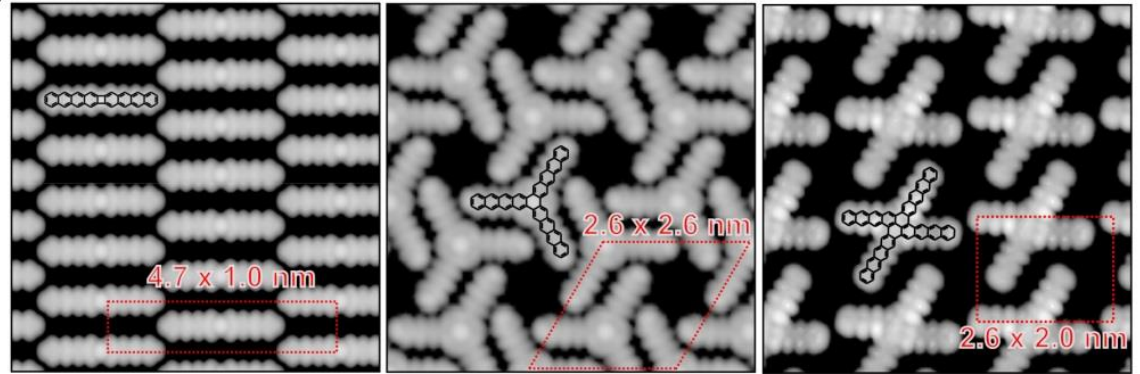

Figure 2.- STM images of linear dimers (2), trimers (3) and tetramers (4) after deposition of 1 onto $\mathrm{Ag}(111)$ held at $430 \mathrm{~K}$. (a) Experimental STM images in phase-pure islands ( $\mathrm{Vs}=-0.1 \mathrm{~V}, \mathrm{I}=1 \mathrm{nA}, 5 \mathrm{~K}$ ). (b) Atomic configuration of the packing of linear dimers (2), trimers (3) and tetramers (4) on Ag(111). (c) DFT simulated STM images for the occupied states region (energy $=-0.5 \mathrm{eV}$ ). For simplicity, the $\mathrm{Br}$ adatoms seen in experiment between the oligomer structures have been omitted.). Dashed red lines indicate the unit cells used for the DFT calculations.

In addition to the three coexisting structures shown in Figure 1, we draw attention to the small circular features flanking all these structures, as clearly seen in Figure 2 a. Due to the nature of the system under study, these can only have two origins: $\mathrm{Br}$ atoms released during the dehalogenation of the precursor monomers, or Ag adatoms released from the step edges. At the substrate temperature at which monomers are deposited ( $430 \mathrm{~K}), \mathrm{Br}$ atoms are known to be still present on the $\mathrm{Ag}(111)$ surface, ${ }_{1}^{20}$ and it seems logical to assign the small round features to $\mathrm{Br}$ atoms. To confirm this hypothesis, we have computed the adsorption energy of $\mathbf{2}$ in the presence of $\mathrm{Br}$ atoms and $\mathrm{Ag}$ adatoms (see Supp. Info. Table S2). These calculations indicate that the adsorption energy is higher by about $850 \mathrm{meV}$ (in absolute terms) for dimers stabilized by $\mathrm{Br}$ atoms compared to $\mathrm{Ag}$ adatoms. Furthermore, an excellent agreement between experimental and simulated STM images is obtained when considering $\mathrm{Br}$ as the stabilizing atoms (see Figure S3 d). This result is not surprising as atomic bromine stabilization of molecular structures into well-ordered islands has been reported previously. ${ }^{21}$ 
In order to rationalize the complex reactions yielding the different structures observed at $430 \mathrm{~K}$, we have calculated the energy barriers involved in the process. The first step is the formation of the activated precursor monomers (aryne) by surface-catalyzed dehalogenation. Figure 3 a shows a STM image of the precursor monomers after deposition on the $\mathrm{Ag}(111)$ surface at room temperature (RT). The asymmetric appearance of the molecules suggests that partial dehalogenation already took place at RT, thus indicating that the dehalogenation proceeds in a two-steps process. The computed dehalogenation energy barriers shown in Figure $3 \mathrm{~b}$ corroborate this result. We observed that the first dehalogenation step exhibits an energy barrier of $600 \mathrm{meV}$ while the second dehalogenation corresponds to a much higher barrier of $900 \mathrm{meV}$. This significant energy barrier difference explains why it is possible to isolate singly-dehalogenated monomers on the surface at RT. It is worthy to mention that partial dehalogenation of bromine-functionalized molecules on $\mathrm{Ag}(111)$ upon deposition at RT has already been reported, ${ }^{22}$ in line with our results. The optimized geometries during the whole dehalogenation sequence are presented in Figure 3 c. Upon deposition, molecules lie flat on the surface (initial state). Once the first bromine atom is detached from the molecule, the carbon radical forms a covalent bond with the surface, and thus forces the molecule to bend towards it (intermediate state). Finally, when the second bromine atom detaches from the molecule, the second radical is also saturated by the bonding to the surface, ending up in a Ag-stabilized aryne molecule which presents a significant curvature. In passing, we note that the nature of ortho-activated aromatic rings (aryne) has been a subject of debate during many decades due to the existence of three possible resonance structures, namely alkyne (one triple bond), cumulene (three double bonds) or biradical (two radicals). ${ }^{23}$ Panels $d$-f in Figure 3 show the calculated charge densities and bond lengths of our aryne/Ag (111) system, respectively. Both charge distribution and bond lengths suggest that the biradical resonance structure is predominant in this case. Given the nature of the substrate, the formation of covalent bonds between the biradical and the $\mathrm{Ag}(111)$ surface (C-Ag bond lengths of $2.18 \AA$ ) is not unexpected, and thus do not seem to contradict previous observations by Pavliček and co-workers who reported on a cumulene resonance structure for an aryne on $\mathrm{NaCl}$, a highly inert substrate ${ }^{23}$ 
(a)

(c)
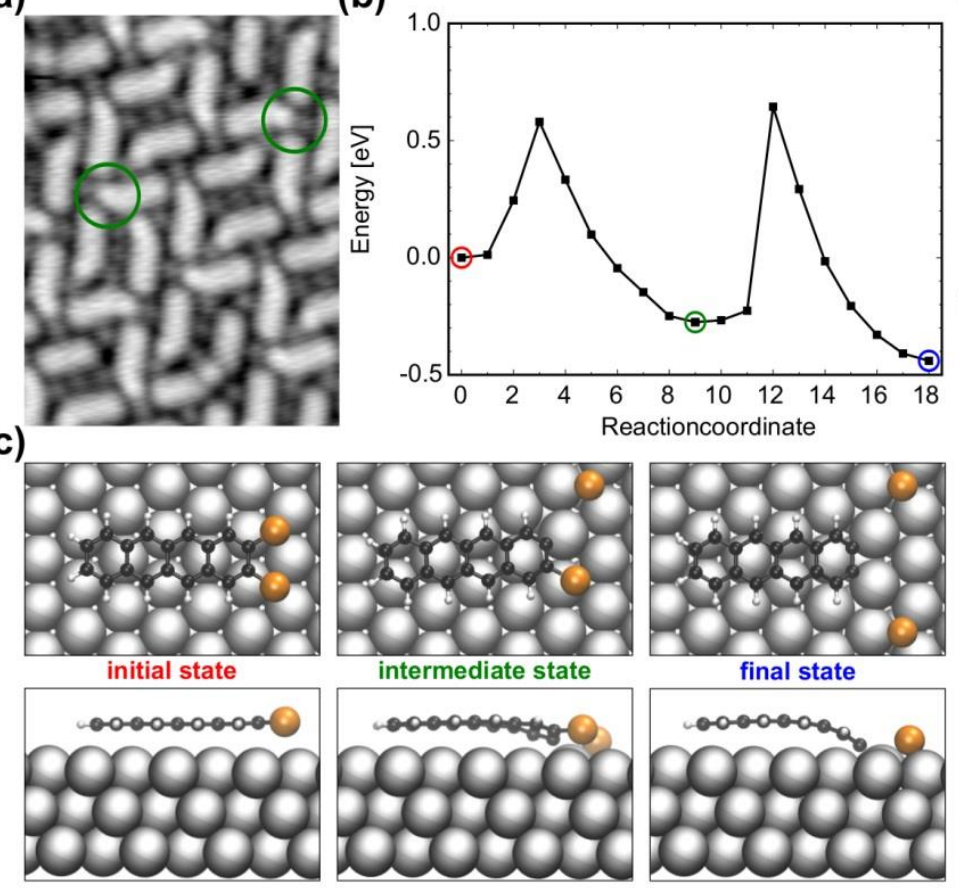

(b)

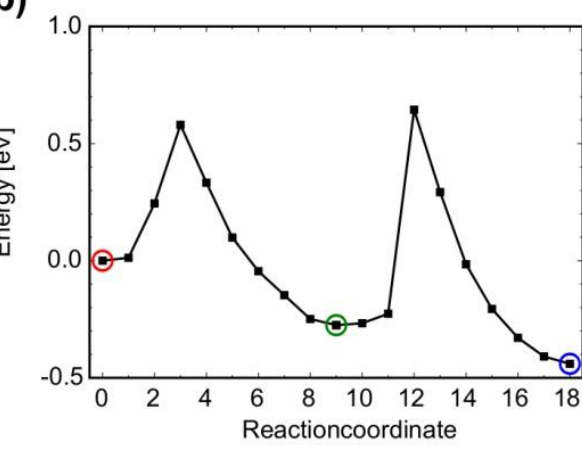

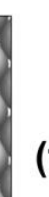

(f)

(d)

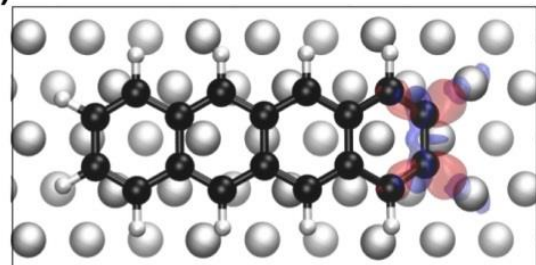

(e)

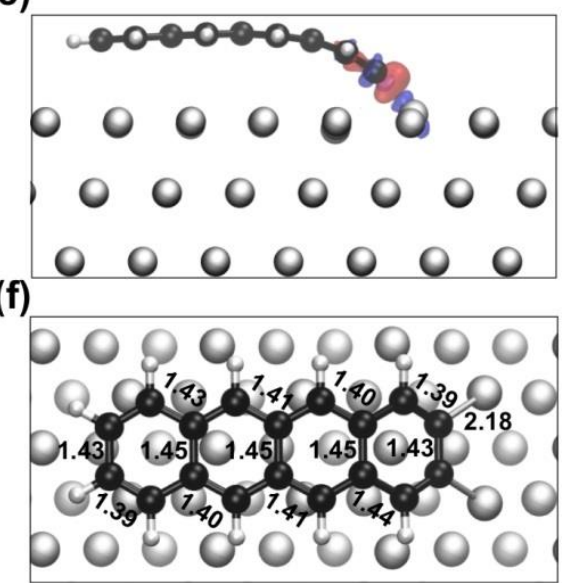

Figure 3.- Dehalogenation of 2,3-dibromotetracene monomer 1 on $\mathrm{Ag}(111)$. (a) STM image of monobromo intermediates (green circles indicating the non-detached $\mathrm{Br}$ species) obtained after deposition of 1 onto $\mathrm{Ag}(111)$ at $\mathrm{RT}(\mathrm{Vs}=-0.3 \mathrm{~V}, \mathrm{I}=0.05 \mathrm{nA}$, $77 \mathrm{~K}$ ). (b) Energy diagram of the dehalogenation process computed using the nudged elastic band (NEB) method. (c) Atomic structures of 2,3-dibromotetracene 1 (red), the monobromo intermediate (green) and the fully dehalogenated biradical product 1' (blue) on Ag(111). (d) Charge density induced by the binding of the biradical 1' to the Ag(111) substrate, with charge accumulation (red) and depletion (blue) regions. The 0.05 a.u isosurfaces are represented in a top view. The induced charge density reveals covalent bonds between the biradical and the $\mathrm{Ag}(111)$ surface, with C-Ag bond lengths of $2.18 \AA$. (e) Side view. (f) C-C bond lengths calculated for the biradical 1' bound to the $\mathrm{Ag}(111)$ surface in its most stable configuration, as illustrated in (d) and (e).

After activation (dehalogenation) of the molecules on the surface, the next step is the formation of the abovementioned structures $\mathbf{2}-\mathbf{4}$ (for the sake of clarity, we will focus on the majority species $\mathbf{2}$ and $\mathbf{3}$ ). Figure 4 summarizes the results of an extensive set of DFT and nudged elastic band (NEB) calculations for the [2+2] and [2+2+2] cycloadditions of biradical 1' on Ag(111) (see Figs. S5 and S6 for further details on the initial orientation of $\mathbf{1}$ ' and its translational and rotational barriers). The upper two panels (Fig. 4 a, b) give the energy profiles along the different reactions paths, whereas the lower panels (Fig. 4 c) show the geometries of the most important configurations found along the reaction paths. Figure 4 a displays the energy barriers for the three paths which can be involved in the formation of the cyclobutadiene-fused tetracene dimer 2. Blue/red/green curves correspond to initial configurations in which two activated monomers ( $\left.\mathbf{1}^{\prime}\right)$ are aligned/shifted/rotated with respect to each other, respectively. In the case of the aligned configuration (blue curve), a single step is necessary in order to form $\mathbf{2}$. This situation requires overcoming a single energy barrier of approximately $500 \mathrm{meV}$ as the final structure is directly formed in a straightforward [2+2] cycloaddition step. However, if the two reactants are not 
directly facing each other but are initially shifted or rotated with respect to each other, two successive aryl-aryl coupling steps are required to reach the final dimer $\mathbf{2}$. In both cases, the barrier to form the first C-C bond is almost negligible, being zero for the rotated configuration (green curve) and $84 \mathrm{meV}$ for the shifted one (red curve). However, the scenario is quite different once the first bond has been formed. For the rotated configuration, a rotation of approximately $120^{\circ}$ is needed in order to create the second bond. This process has an energy barrier of $737 \mathrm{meV}$. This is much lower than the one needed to flip one of the monomers around the $\sigma$ bond in the shifted configuration, which is 1407 $\mathrm{meV}$. One has to take into account that the initial energy barrier for the complete dehalogenation is around $900 \mathrm{meV}$, which, in an adiabatic situation, is sufficient to subsequently overcome the barrier in the aligned and rotated configurations, but not in the shifted one. This energy difference thus rationalizes why some shifted dimers (2,2'-bitetracenyl) can be found on the surface.

Figure $4 \mathrm{~b}$ presents the results of a similar analysis for the formation of $3 \mathrm{via}[2+2+2]$ cycloaddition. In this case, just a single path has been considered: first, the formation of a rotated dimer and then the addition of a third biradical $\mathbf{1}$ ' to form the final tetracene trimer $\mathbf{3}$. For this situation, the highest energy barrier corresponds to the first aryl-aryl coupling step that yields the rotated (singly bound) dimer (264 $\mathrm{meV})$. In contrast, the energy required to add a third monomer to complete the cyclotrimerization is only $107 \mathrm{meV}$. Clearly, both values are well below the energy needed to fully dehalogenate the starting monomer 1, so no thermodynamical limitation should get in the way of the formation of trimers if we again assume adiabatic conditions. This prediction is in accordance with the observation of trimers at $430 \mathrm{~K}$ (see Table S3 in Supp. Info. for details on the energy diagrams).

We have not explicitly computed energy barriers for the formation of tetramers 4 from four 1 '. However, consideration of the individual steps involved in the different possible pathways to 4 (see Figure S4) reveals that these are very similar to the elementary steps involved in the dimerization and trimerization processes discussed above. It is thus reasonable to expect an overall energy barrier of similar amplitude compared to the one of the dimerization and trimerization paths. This hypothesis is also suggested by the experimental observation that all three products 2, 3 and $\mathbf{4}$ coexist after activation at $430 \mathrm{~K}$ (Fig. 1). 
(a)

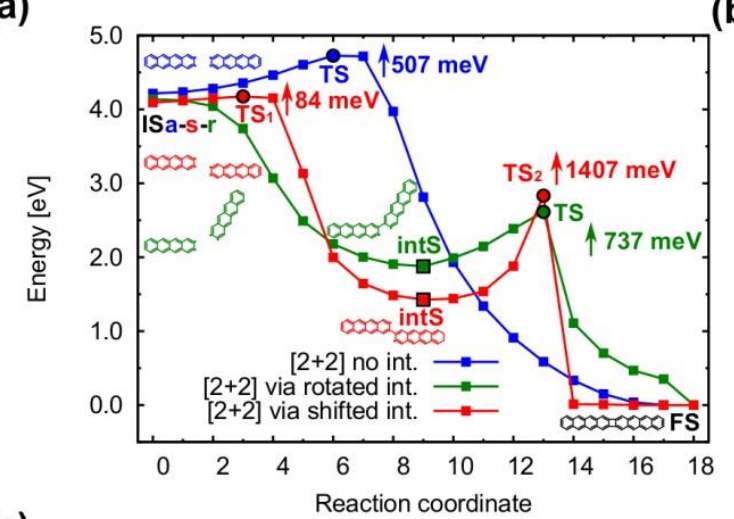

(c)
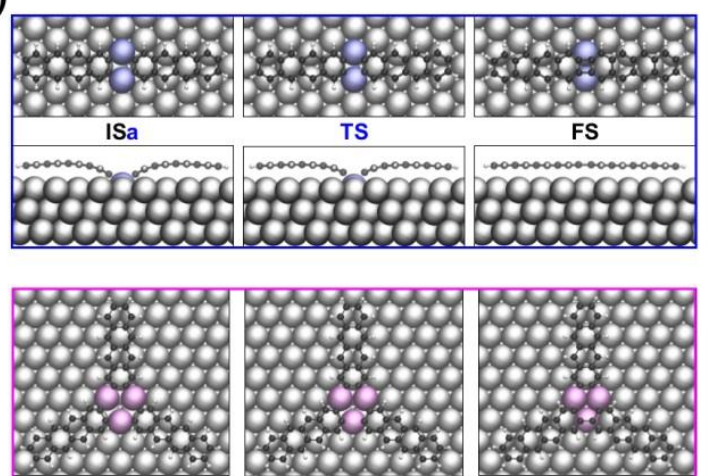

IS
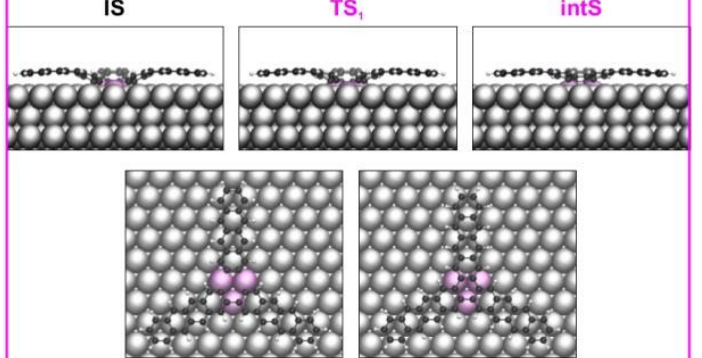

TS

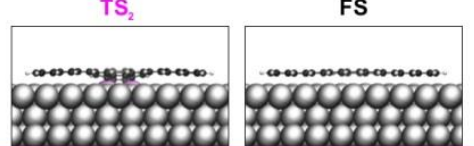

(b)
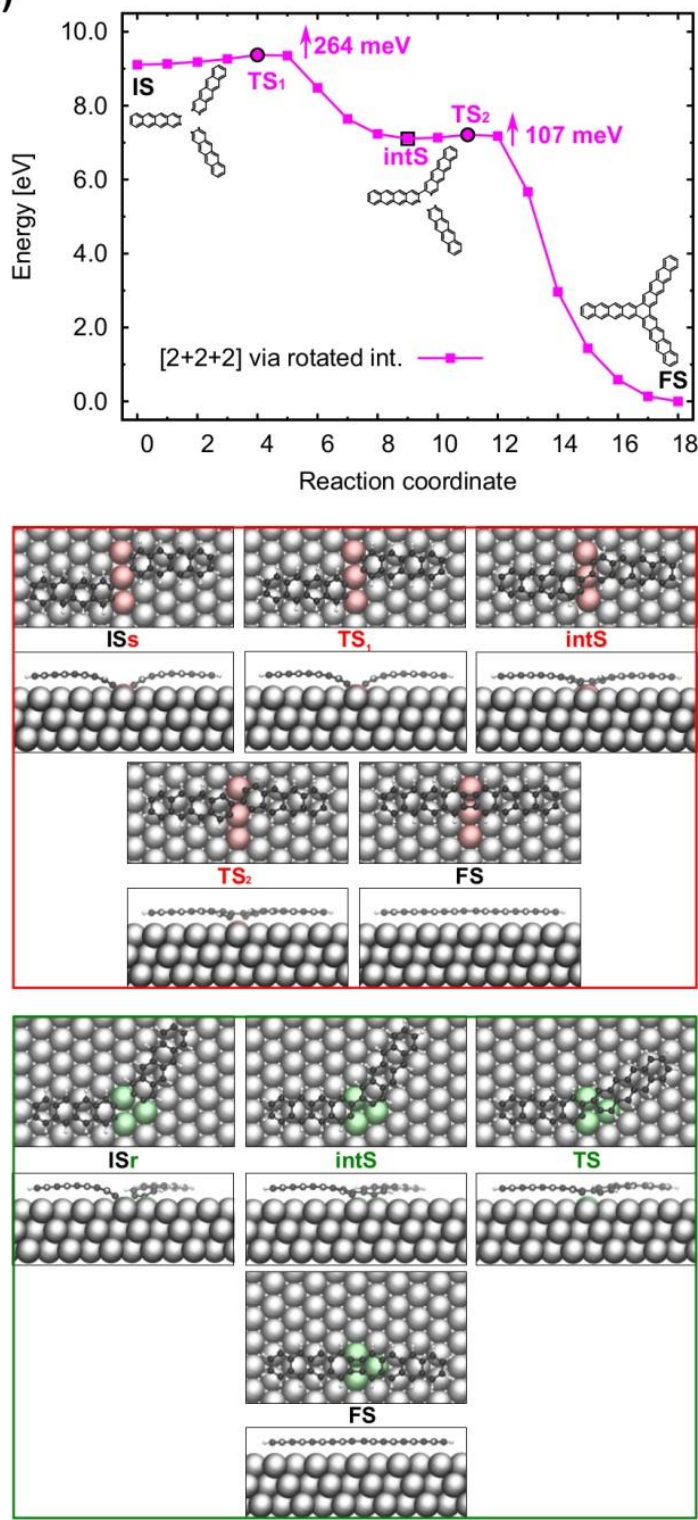

Figure 4.- Dimerization and trimerization of 1' via cycloaddition on $\mathrm{Ag}(111)$ from NEB calculations. (a) Energy diagram for the dimerization $([2+2]$ cycloaddition) from different initial configurations of two 1 ' molecules: initial state for which the two reactants are aligned (ISa, blue line), initial state for which the reactants are axially offset/shifted (ISs, red line), and initial state for which the two reactants are rotated by $120^{\circ}$ (ISr, green line) with respect to each other. Transition states (TS) and intermediate states (intS) are indicated with black circles and squares, respectively. (b) Energy diagram for the trimerization of 1' $([2+2+2] \mathrm{cy}-$ cloaddition, magenta line). (c) Top and side views of the initial state (IS), transition state (TS), intermediate state (intS) and final state (FS) geometries for the different reaction pathways, with color coding of the frames analogous to the one of the energy profiles in (a) and (b). Highlighted Ag atoms indicate substrate atoms directly involved in the reaction.

The above discussions prompt the question whether some selectivity with respect to the formation of dimers, trimers, or tetramers can be obtained by the appropriate control of the reaction conditions. From the computational point of view, this appears questionable since the barriers to the [2+2] and $[2+2+2]$ cycloadditions are very similar (Fig. 4) and lower than the barrier to activate (i.e., dehalogenate) the starting monomers $\mathbf{1}$. In a more or less adiabatic situation, one would thus expect $\mathbf{1}$ ' to proceed 
immediately to the final products $\mathbf{2}, \mathbf{3}$, and $\mathbf{4}$, with limited selectivity regarding the different products. In other words, dehalogenation is the overall rate limiting step with respect to all reaction pathways. Indeed, we could not find experimental conditions that would provide high selectivity toward one or the other reaction product. This is illustrated in Figure S7 where the impact of substrate temperature is found to be limited. Although a trend towards enhanced tetramerization at lower temperatures and enhanced dimerization at higher temperatures can be discerned, we could not exclusively and selectively produce one or the other structure. There can be several reasons for this finding since many factors are involved, including parameters that are difficult to control such as kinetics, diffusion of intermediates on the surface or monomer purity. Further studies, including other substrates, are needed to clarify the question whether selective product formation can be accomplished for the present onsurface cycloadditions.

On-surface synthesis can thus provide access to new reaction pathways under UHV conditions and allow a straightforward in situ characterization at molecular level. Compared to surface chemistry, advantages of solution synthesis are a much broader choice of experimental conditions and structure proof by the breadth of established instrumental analysis. It appeared appropriate to explore whether products 2, 3, and $\mathbf{4}$ obtained via on-surface synthesis could also be achieved via conventional solution synthesis and whether product selectivity would be possible under such reaction conditions. Obviously, while the on-surface synthesis method required sublimation of the starting compounds, solution chemistry needed sufficient solubility. In this case, TIPS-ethynyl groups were attached to the tetracene (1-TIPS, Fig. S1), providing good solubility in common organic solvents. Interestingly, the trimers (trimer-TIPS) were selectively achieved in moderate yield (38 \%) through Yamamoto coupling at $100{ }^{\circ} \mathrm{C}$ in toluene/DMF. Different from the surface results, we could neither observe the dimer 2 nor the tetramer 4 (Fig. 1). This outcome is consistent with a previous report by Bunz's group. ${ }^{24}$ The resulting Trimer-TIPS has good solubility in organic solvents, such as dichloromethane (DCM), tetrahydrofuran (THF) and toluene. This allowed a full spectroscopic characterization including optical properties. The UV-Vis absorption spectra of tetracene and trimers in DCM solution are compared in Fig S1. The absorption maximum of Trimer-TIPS shows a significant bathochromic shift with respect to that of tetracene, with an absorption maximum at $477 \mathrm{~nm}$ and three other shoulder peaks at 591, 592 and 644 $\mathrm{nm}$, respectively. Thus, the optically determined HOMO-LUMO gap of Trimer-TIPS is determined from the onsets of their UV-Vis absorption maximum as $1.78 \mathrm{eV}$ which certainly reflects the extended conjugation of Trimer-TIPS after the cyclization. It is unclear whether the differences in product formation of solution and on-surface chemistry are also due to the substitution within the former case.

\section{Conclusions}


We have demonstrated a new strategy for the on-surface formation of covalent C-C bonds via formal cycloaddition of precursor monomers that are halogen functionalized at the ortho $(2,3)$ positions of arenes. With our choice of a 2,3-dibromotetracene precursor, this approach allows for the formation of tetracene linear dimers, cyclo-trimers and X-shaped tetramers. This product variety is possible thanks to two different coupling motifs, namely the formation of four-membered rings (cyclobutadiene units) via a [2+2] cycloaddition and the formation of six-membered rings via a $[2+2+2]$ cycloaddition, respectively. The formation of these structures has been monitored by STM and rationalized by extensive DFT calculations, which has allowed us to develop a comprehensive mechanistic description of the observed reactions. Furthermore, we find that the 2,3-dibromotetracene precursor can also undergo the selective formation of cyclo-trimers under wet chemistry conditions. ortho-Halogen functionalization and the resulting cycloadditions provide a new tool to the emerging field of on-surface synthesis, and a potential pathway to the synthesis of intriguing one-dimensional structures such as biphenylenebridged acene ribbons.

\section{Methods}

Experiments have been carried out in two ultra-high vacuum system (base pressure below $10^{-10} \mathrm{mbar}$ ), one of them equipped with a LT-STM ( $5 \mathrm{~K}$, Scienta Omicron, $\mathrm{GmbH})$ and the other one with a VT-STM (Scienta Omicron, GmbH). Ag(111) single crystals (Surface Preparation Laboratory, SPL) were cleaned by repeated $\mathrm{Ar}+$ sputtering $(1.0 \mathrm{kV})$ and annealing $(750 \mathrm{~K})$ cycles until judged clean by STM. Orthodibromotetracene molecules were thermally evaporated from a 3-fold Kentax evaporator or from a 6fold ORMA evaporator (Mantis Deposition), stabilized in temperature through a PID controller. A deposition rate of approximately $1 \AA /$ min (determined by a quartz micro-balance) was used for deposition (deposition temperatures of $400 \mathrm{~K}$ and $440 \mathrm{~K}$ for Kentax and ORMA, respectively). STM images were recorded in constant current mode. Unless otherwise stated, all STM images were recorded at $5 \mathrm{~K}$. All sample preparation temperatures expressed throughout the manuscript were measured with a pyrometer focused on the sample surface and aligned perpendicular to it (Optris, emissivity $\varepsilon=0.1$ ).

$A b$ initio plane-wave Density Functional Theory (DFT) calculations were performed using the VASP software package ${ }^{25,26}$ equipped with the projector augmented-wave method for core electron-ion interactions..$^{27}$ The exchange-correlation interaction is considered in the GGA using the Perdew-BurkeEnzerhof functional. ${ }^{28}$ Van der Waals ( $\mathrm{vdW}$ ) interactions are included within a DFT-D2 approach of Grimme, $^{29}$ as implemented in VASP 5.2. We have used the values of $24.67,1.75,0.14$ and 12.47 J.nm $6 / \mathrm{mol}$ for the dispersion coefficients $C_{6}$ and the values of $1.639,1.452,1.001$ and $1.749 \AA$ for the van der Waals radius $\mathrm{R}_{0}$ of $\mathrm{Ag}, \mathrm{C}, \mathrm{H}$ and $\mathrm{Br}$ atoms, respectively. A cutoff energy of $400 \mathrm{eV}$ is chosen for the plane-wave basis set using a fine k-point sampling within the Monkhorst-Pack scheme ${ }^{30}$ and all the atoms were relaxed until all residual forces were below $0.02 \mathrm{eV} / \AA$ using the conjugate gradient algo- 
rithm as implemented in VASP 5.2 .

The Ag(111) surface was modelled by a periodic slab geometry constructed from the GGA PBE+D2 optimized Ag bulk, leading to a lattice constant of $4.142 \AA$ (4.090 $\AA$ for the experimental one) and each unit cell contains three layers. In order to avoid interactions between the slab and its periodic images along the surface normal, a $25 \AA$ vacuum region was added. The shape and the lateral size of the slab is dependent on the type of molecules adsorbed on the $\mathrm{Ag}(111)$ surface: a rectangular slab of $3.55 \mathrm{x}$ $1.46 \mathrm{~nm}^{2}$ was used for the activated monomers (with a k-point sampling of $2 \times 2 \times 1$ ) as shown in Fig. 3; a rectangular slab of $4.69 \times 1.01 \mathrm{~nm}^{2}$ was used for linear dimers (with a k-point sampling of $1 \times 5 \times 1$ ) containing 2 close packed linear dimers as shown in Fig. 2; an hexagonal slab of $2.64 \times 2.64 \mathrm{~nm}^{2}$ was used for trimers (with a k-point sampling of $2 \times 2 \times 1$ ) containing 2 closed packed trimers as shown in Fig. 2, and finally, a rectangular slab of $2.64 \times 2.03 \AA^{2}$ was used for tetramers (with a k-point sampling of $2 \times 2 \times 1$ ) as shown in Fig. 2 . In all geometry optimizations, the atoms of the adsorbed molecules and the top layer atoms of the substrate were allowed to relax, the other substrate atoms were kept fixed at their bulk positions (test calculations performed including an additional Ag layer allowed to relax did not show major differences).

Reaction energy barrier calculations have been performed using the Nudge Elastic Band method in the gamma point approximation as implemented in VASP 5.2. ${ }^{31}$ The number of images for each calculation is 8 and all the atoms were relaxed in NEB calculations until all residual forces were below $0.05 \mathrm{eV} / \AA$ using the RMM-DIIS quasi-Newton algorithm as implemented in VASP 5.2.

\section{Corresponding Authors}

cssanchez@icmm.csic.es

roman.fasel@empa.ch

* C.S.S. and A.N. are equally contributing authors

${ }^{+}$Current address:

\# Current address: Faculty of Materials Science and Engineering, Kunming University of Science and Technology, 650000 Kunming, China.

\section{Acknowledgements}

We are grateful for financial support from the Swiss National Science Foundation, the Office of Naval Research BRC program, and the European Commission Graphene Flagship (No. CNECT-ICT-604391). CSS is grateful to Ministerio de Economía y Competitividad for financial support via the Juan de la Cierva Incorporación grant (IJCl-2014-19291). We thank Jia Liu for preliminary experiments. 
Supporting Information Available: Monomer synthesis and characterization, DFT study on the most stable configuration and diffusion for tetracene biradicals, Gibbs adsorption free energy of linear dimers, adsorption energies in the presence of $\mathrm{Ag}$ and $\mathrm{Br}$ adatoms, proposed reaction schemes, energy barriers during dimer and trimer formation and influence of substrate temperature during monomer deposition. This material is available free of charge via the Internet at http://pubs.acs.org.

\section{References}

1. Nishiwaki, N. Methods and applications of cycloaddition reactions in organic syntheses. (Wiley-VCH, 2014).

2. Zaragoza Dörwald, F. Organic synthesis on solid phase: supports, linkers, reactions. (Wiley-VCH, 2000).

3. Pérez, D., Peña, D. \& Guitián, E. Aryne Cycloaddition Reactions in the Synthesis of Large Polycyclic Aromatic Compounds: Synthesis of Large Polycyclic Aromatic Compounds. Eur. J. Org. Chem. 2013, $5981-6013$ (2013).

4. Alcaide, B., Almendros, P. \& Aragoncillo, C. Exploiting [2+2] cycloaddition chemistry: achievements with allenes. Chem Soc Rev 39, 783-816 (2010).

5. Shen, Q., Gao, H.-Y. \& Fuchs, H. Frontiers of on-surface synthesis: From principles to applications. Nano Today 13, 77-96 (2017).

6. Bebensee, F. et al. On-Surface Azide-Alkyne Cycloaddition on Cu(111): Does It 'Click' in Ultrahigh Vacuum? J. Am. Chem. Soc. 135, 2136-2139 (2013).

7. Díaz Arado, O. et al. On-Surface Azide-Alkyne Cycloaddition on Au(111). ACS Nano 7, 8509-8515 (2013).

8. Liu, J., Ruffieux, P., Feng, X., Müllen, K. \& Fasel, R. Cyclotrimerization of arylalkynes on Au(111). Chem. Commun. 50, 11200 (2014).

9. Zhou, H. et al. Direct Visualization of Surface-Assisted Two-Dimensional Diyne Polycyclotrimerization. J. Am. Chem. Soc. 136, 5567-5570 (2014). 
10. Xiang, F. et al. Cyclotrimerization-Induced Chiral Supramolecular Structures of 4Ethynyltriphenylamine on Au(111) Surface. Chem. - Eur. J. 21, 12978-12983 (2015).

11. Yang, B. et al. Synthesis of Surface Covalent Organic Frameworks via Dimerization and Cyclotrimerization of Acetyls. J. Am. Chem. Soc. 137, 4904-4907 (2015).

12. Fesser, P. et al. Visualizing the Product of a Formal Cycloaddition of 7,7,8,8-Tetracyano-pquinodimethane (TCNQ) to an Acetylene-Appended Porphyrin by Scanning Tunneling Microscopy on $\mathrm{Au}(111)$. Chem. - Eur. J. 17, 5246-5250 (2011).

13. Cirera, B. et al. Thermal selectivity of intermolecular versus intramolecular reactions on surfaces. Nat. Commun. 7, 11002 (2016).

14. Lindner, R. et al. Substrate Templating Guides the Photoinduced Reaction of C60 on Calcite. Angew. Chem. Int. Ed. 53, 7952-7955 (2014).

15. Liu, M. et al. Graphene-like nanoribbons periodically embedded with four- and eight-membered rings. Nat. Commun. 8, 14924 (2017).

16. de Oteyza, D. G. et al. Direct Imaging of Covalent Bond Structure in Single-Molecule Chemical Reactions. Science 340, 1434-1437 (2013).

17. Cui, P. et al. Carbon Tetragons as Definitive Spin Switches in Narrow Zigzag Graphene Nanoribbons. Phys. Rev. Lett. 116, (2016).

18. On-Surface Synthesis. (Springer International Publishing, 2016).

19. Luo, J. \& Hart, H. Linear acene derivatives. New routes to pentacene and naphthacene and the first synthesis of a triptycene with two anthracene moieties. J. Org. Chem. 52, 4833-4836 (1987).

20. Goddard, P. J., Schwaha, K. \& Lambert, R. M. Adsorption-desorption properties and surface structural chemistry of bromine on clean and sodium-dosed Ag(111). Surf. Sci. 71, 351-363 (1978).

21. Simonov, K. A. et al. From Graphene Nanoribbons on $\mathrm{Cu}(111)$ to Nanographene on $\mathrm{Cu}(110)$ : $\mathrm{Criti}-$ cal Role of Substrate Structure in the Bottom-Up Fabrication Strategy. ACS Nano 9, 8997-9011 (2015). 
22. Eichhorn, J. et al. On-surface Ullmann polymerization via intermediate organometallic networks on Ag(111). Chem. Commun. 50, 7680-7682 (2014).

23. Pavliček, N. et al. On-surface generation and imaging of arynes by atomic force microscopy. Nat. Chem. 7, 623-628 (2015).

24. Rüdiger, E. C., Porz, M., Schaffroth, M., Rominger, F. \& Bunz, U. H. F. Synthesis of Soluble, AlkyneSubstituted Trideca- and Hexadeca-Starphenes. Chem. - Eur. J. 20, 12725-12728 (2014).

25. Kresse, G. \& Furthmüller, J. Efficiency of $a b$-initio total energy calculations for metals and semiconductors using a plane-wave basis set. Comput. Mater. Sci. 6, 15-50 (1996).

26. Kresse, G. \& Furthmüller, J. Efficient iterative schemes for ab initio total-energy calculations using a plane-wave basis set. Phys. Rev. B 54, 11169 (1996).

27. Kresse, G. \& Joubert, D. From ultrasoft pseudopotentials to the projector augmented-wave method. Phys. Rev. B 59, 1758 (1999).

28. Perdew, J. P., Burke, K. \& Ernzerhof, M. Generalized gradient approximation made simple. Phys. Rev. Lett. 77, 3865 (1996).

29. Grimme, S. Semiempirical GGA-type density functional constructed with a long-range dispersion correction. J. Comput. Chem. 27, 1787-1799 (2006).

30. Monkhorst, H. J. \& Pack, J. D. Special points for Brillouin-zone integrations. Phys. Rev. B 13, 51885192 (1976).

31. Jónsson, H., Mill, G. \& Jacobsen, K. W. Nudged elastic band method for finding minimum energy paths of transitions. in Classical and Quantum Dynamics in Condensed Phase Simulations 385 (World Scientific, 1998). 